\title{
ON THE HOLONOMY GROUPS OF LINEAR CONNECTIONS
}

\author{
JUN-ICHI HANO and HIDEKI OZEKI
}

In this note we show in $\S 1$, as the main result, that any connected Lie subgroup of the general linear group $G L(n, R)$ can be realized as the holonomy group of a linear connection, i.e. the homogeneous holonomy group of the associeted affine connection, defined on an affine space of dimension $n(n \geqslant 2)$.

In the case of Riemannian connections it is known that the restricted homogeneous holonomy group is always closed in $G L(n, R)$. Our result shows that this is no longer true for general linear connection. We shall furthermore give an example of a linear connection without torsion whose restricted holonomy group is not closed $(\S 2)$.

Throughout this note we use the results, together with the terminology of W. Ambrose and I. M. Singer in their paper "A theorem on holonomy groups" (Trans. Amer. Math. Soc. vol. 75, 1953).

$\mathrm{K}$. Nomizu extends our result in $\S 1$ to the case of general connections on principal fiber bundles (In this journal).

1. Let $M$ be an affine space of dimension $n$ and $\left(x^{i}\right)$ a system of cartesian coordinates. Then the bundle of frames $F(M)$ of $M$ has a system of coordinates $\left(x^{i}, X_{k}^{j}\right)$, such that the $n$ vectors of a frame are given by $\sum_{k=1}^{n} X_{j}^{k} \frac{\partial}{\partial x^{k}}(j=1$, $\ldots, n)$, where the determinant of $\left(X_{j}^{k}\right)$ is not zero. We denote by $x$ a point in $M$ with the coordinate $\left(x^{i}\right)$ and by $(x, X)$ a point in $F(M)$ with the coordinate $\left(x^{i}, X_{k}^{j}\right)$, especially by $(x, \delta)$ a point with the coordinate $\left(x^{i}, \delta_{k}^{j}\right)$.

The structure group of the bundle $F(M)$ is the general linear group $G L(n, R)$ and an element $Y=\left(Y_{k}^{j}\right)$ of $G L(n, R)$ defines the right translation $R_{Y}:\left(x^{i}, X_{k}^{j}\right) \rightarrow\left(x^{i}, \sum_{e=1}^{n} X_{e}^{j} Y_{k}^{e}\right)$ of $F(M)$.

A linear connection on $M$ is defined by the so-called coefficients of linear connection $\Gamma_{j k}^{i}(x)$, where $n$ differentiable functions $\Gamma_{j k}^{i}(x)$ on $M$ can be arbitrarily chosen.

Received December 28, 1955. 
Then the $n$-dimensional subspace of the tangent space at $(x, X)$ of $F(M)$ spanned by

$$
\mathfrak{X}_{i(x, X)}=\partial_{\partial x^{i}(x, X)}-\sum_{j, k=1}^{n} I_{j k}^{i}(x) d R_{X}\left(\frac{\partial}{\partial X_{k}^{j}(x, \delta)}\right) \quad(i=1, \ldots, n)
$$

is the horizontal subspace associated to the connection. And the $n$ differential forms

$$
\omega_{k(x, X)}^{j}=\sum_{e=1}^{n}\left(X^{-1}\right)_{e}^{j}\left(d X_{k}^{e}+\sum_{p, q=1}^{n} \Gamma_{p q}^{e}(x) d x^{p} X_{k}^{q}\right)
$$

define the $(g l(n, R)$-valued $)$ connection form $\omega$. According to W. Ambrose and I. M. Singer the structure equation is given by

$$
d \omega=-\frac{1}{2}[\omega, \omega]+\Omega
$$

where $\Omega$ is the curvature form.

When the dimension of $M$ is equal to 1 , the holonomy group of any linear connection on $M$ consists of only the neutral element. Hereafter we assume that the dimension of $M$ is $\geqslant 2$.

We take an arbitrary connected Lie subgroup $G$ in the general linear group $G L(n, R)$. Let $\&$ be the Lie subalgebra associated to $G$ in the general linear Lie algebra $g \cdot(n, R)$, and let $V_{i}=\left(v_{i k}^{j}\right)(i=1, \ldots, m)$ be a basis of $g$, where $m$ is the dimension of $\mathrm{g}$.

We take arbitrary $m$ positive numbers $a_{1}<a_{2}<\ldots<a_{m}$, and construct $m$ functions

$$
g_{\alpha}(u)=\int_{0}^{u} \prod_{\alpha \neq \beta}\left(t-a_{\beta}\right) d t \quad(\alpha=1, \ldots, m)
$$

Then the function $g_{\alpha}(u)(\alpha=1, \ldots, m)$ is an analytic function of the real variable $u$, and its derivative $d g_{\alpha} / d u$ is zero at $u=a_{\beta}$ if $\beta \neq \alpha$ and is $c_{\alpha}=\prod_{\alpha \neq \beta}\left(a_{\alpha}-a_{3}\right) \neq 0$ at $u=a_{\alpha}$.

We now define a linear connection on $M$, whose holonomy group is $G$, as follows :

$$
\begin{aligned}
& \Gamma_{1 k}^{i}(x)=\sum_{\alpha=1}^{m} f_{\alpha}(x) v_{\alpha k}^{i}, \quad \text { where } f_{\alpha}(x)=g\left(x^{2}\right), \\
& \Gamma_{j k}^{i}(x)=0 \text { for } \quad j \geq 2 .
\end{aligned}
$$

Then the horizontal vector fields are given by 


$$
\begin{aligned}
& \mathfrak{X}_{1(x, X)}=\frac{\partial}{\partial x^{1}(x, x)}-\sum_{\alpha=1}^{m} f_{\alpha}(x) \cdot V_{\alpha(x, X)}, \\
& \mathfrak{X}_{j(x, x)}=\frac{\partial}{\partial x^{j}(x, X)} \quad \text { for } \quad j \geqslant 2,
\end{aligned}
$$

where $V_{a(x, X)}=d R_{X}\left(\sum_{i, k=1}^{n} v_{\alpha k}^{i} \frac{\partial}{\partial x_{k(x, \delta)}^{i}}\right)$.

We shall prove that the holonomy group $\Phi$ with the reference point $(0, \delta)$ of our linear connection defined above is $G$. First we prove that $\Phi$ containes $G$. The curve $\sigma(t)=(0, t, 0 \ldots 0, \delta)$ through the reference point $(0, \delta)$ in $F(M)$, whose tangent vector at $\sigma(t)$ is $\mathfrak{X}_{2 \sigma(t)}$, is a horizontal curve. Then $\Omega\left(\mathfrak{X}_{1}, \mathfrak{X}_{2}\right)_{\sigma(t)}$, is contained in $\mathfrak{g}$ according to W. Ambrose and I. M. Singer's theorem. From the structure equation it is easily seen that

$$
\Omega\left(\mathfrak{X}_{1}, \mathfrak{X}_{2}\right)=-\omega\left(\left[\mathfrak{X}_{1}, \mathfrak{X}_{2}\right]\right),
$$

as $\mathfrak{X}_{1}, \mathfrak{X}_{2}$ are horizontal vector fields. And we have from our definition

$$
\left[\mathfrak{X}_{1}, \mathfrak{X}_{2}\right]_{\sigma\left(a_{\alpha}\right)}=\sum_{\beta=1}^{m}\left(\frac{\partial f_{\beta}}{\partial x^{2}}\right)_{\left(0, a_{\alpha}, 0, \ldots, 0\right)} V_{\beta \jmath\left(a_{\alpha}\right)}=c_{\alpha} \cdot V_{\alpha s\left(a_{\alpha}:\right.}
$$

and hence $\Omega\left(\mathfrak{X}_{1}, \mathfrak{X}_{2}\right)_{r\left(a_{\alpha}\right)}=c_{\alpha} V_{\alpha}$ for any $\alpha=1,2, \ldots, m$. This shows that $n$ is contained in the Lie subalgebra associated to $\mathscr{D}$, and $G$ is contained in $\mathscr{D}$.

Next we prove that $\Phi$ is contained in $G$. The subset $P=\left\{(x, X) ;\left(X_{k}^{\prime}\right) \in G\right\}$ in $F(M)$ can be identified with the direct product $M \times G$ and by this identification it becomes a submanifold of $F(M)$. It is clear from our definition that the horizontal subspace at each point in $P$ is contained in the tangent vector space of $P$ then the submanifold in $F(M)$ consisting of all points which can be joined with the reference point $(0, \delta)$ by a horizontal curve is contained in $P$. And for any two horizontal vector fields $\mathfrak{x}, \mathfrak{?}$

$$
\Omega(\mathfrak{X}, \mathfrak{Y})_{(x, X)}=-\omega([\mathfrak{X}, \mathfrak{Y}])_{(x, x)}
$$

is contained in $\mathscr{B}$ if $(x, X) \in P$. Because $[X \mathbb{X}, \mathfrak{Y}]_{(x, X)}$ is contained in the tangent space of $P$ if $(x, X) \in P$. Therefore according to W. Ambrose and I. M. Singer's theorem the holonomy group $\Phi$ is contained in $G$. Thus we have completed the proof of the following theorem;

Any connected Lie subgroup of the general linear group $G L(n, R)$ can be the holonomy group of some linear connection on $M$ of dimension $n(n \geq 2)$.

2. The theorem in $\S 1$ shows that the restricted holonomy group of a linear 
connection is not necessarily closed subgroup of $G L(n, R)$. But the connection in $\S 1$ has the non-zero torsion tensor. We shall give an example which shows that even if the torsion tensor is zero the restricted holonomy group of a linear connection is not necessary closed.

Let $M$ be a six dimensional affine space. We define a linear connection as follows ;

$$
\begin{aligned}
& \Gamma_{1 k}^{i}(x)=0, \\
& \left(\Gamma_{2 k}^{i}(x)\right)=\left(\begin{array}{cccccc}
0 & 0 & & & & \\
0 & 0 & & & & \\
& & 0 & x^{1} & & \\
& & -x^{1} & 0 & & \\
& & & & 0 & \sqrt{2} x^{1} \\
& & & & -\sqrt{2} x^{1} & 0
\end{array}\right),
\end{aligned}
$$

and for $j$ 로 3

$$
\Gamma_{j k}^{i}(x)=0 \quad \text { if } \quad k \neq 2, \quad \Gamma_{j 2}^{i}(x)=\Gamma_{2 j}^{i}(x) .
$$

Then of course the torsion tensor of this connection is zero and it is immediately seen the holonomy group is a non closed subgroup consisting of elements

$$
\left(\begin{array}{cccccc}
1 & 0 & & & & \\
0 & 1 & a_{1} & a_{2} & a_{3} & a_{4} \\
& & \cos \theta & \sin \theta & & \\
& -\sin \theta & \cos \theta & & \\
& & & & \cos \sqrt{2} \theta & \sin \sqrt{2} \theta \\
& & & & -\sin \sqrt{2} \theta & \cos \sqrt{2} \theta
\end{array}\right),
$$

where $a_{1}, \ldots, a_{4}$ and $\theta$ are arbitrary real numbers.

Mathematical Institute

Nagoya University 